\title{
Correspondence
}

\section{Marking of excision margins of discrete skin lesions}

We would like to draw the attention of readers to a method of marking the lateral margins of discrete skin lesions which is of particular value in lesions such as malignan melanomas or basal cell carcinomas. The normal skin at the periphery of the ellipse of skin surrounding the lesion is marked with the tip of a scalpel previously dipped in Indian ink or equivalent dye and the tip of the scalpel is then run around one half of the perimeter of the skin biopsy specimen as illustrated. Following this, transverse and cruciate blocks of the lesion are taken, as required. This method is much quicker and more convenient than painting lateral cutaneous margins with ink and is readily applicable to excision biopsy specimens of skin lesions in nearly all situations.

DN POLLER Department of Histopathology, Great Royal Hospital Great Western Road,

L GROTTICK Department of Histopathology City Hospital, Hucknall Road

Nottingham, NG5 $1 P B$

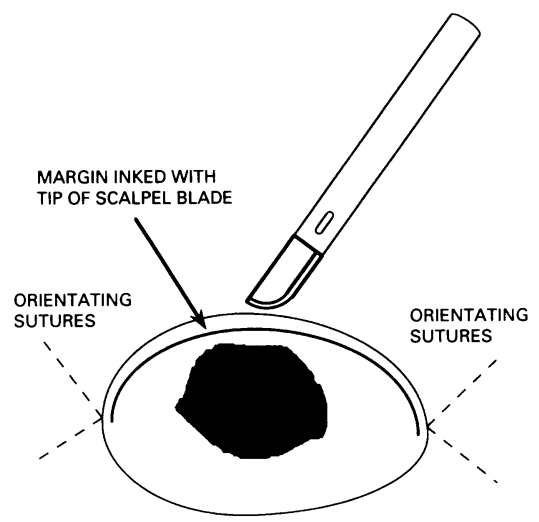

\section{Market testing clinical services}

Recently, the NHS management executive published a White Paper outlining guidelines for market testing services. ${ }^{1}$ This list was noticeably devoid of mention of clinical services, but at least one trust has been in the news for its attempt to contract out its pathology to a private venture. ${ }^{2}$

Clinical services, like radiology and pathology in particular, are often perceived as areas where contracting out part or all of the service is a strong possibility. This per- ception has been boosted by the announcement of the go ahead to ventures between NHS institutions and the private sector. The establishment of larger health authorities has also created single purchasing authorities, with more than one provider within their boundaries, and increased likelihood of rationalisation of provider services. Pathology is a much softer target than radiology; many of those who are not in contact with the way it is practised and delivered perceive the service as relying heavily on the analysis of biological specimens, which does not necessarily have to be carried out on site. The need for local professional input into the different phases of the analytical process is seen as of secondary nature. But there are few specific guidelines available to local managers and users to help them with the assessment of the standards of quality that need to be maintained for effective delivery of service.

The Clinical Pathology Accreditation (CPA) UK scheme has been in operation for over a year. Participation in the scheme is voluntary and it is too early to assess its effect on the practice of pathology in the United Kingdom. The scheme's main aim is also to ensure good laboratory practice rather than to address local standards of service delivery. The Scottish Office has published guidelines for the specification of laboratory service for general managers, but these are too general and need a good deal of fine tuning to meet local requirement.

It is often presumed by users that results produced by laboratories are accurate and precise; taking part in a recognised quality assurance scheme is taken on its face value to indicate acceptable performance. However, information on poor performers is not available, because of the sensitivity and confidentiality of the results; though extreme deviations are reported to steering committees.

While numerical quality assurance values could be assessed, assessment of other aspects of the delivery of an effective diagnostic service is not so straightforward. Apart from turnround time, many aspects of service provision tend to rely on the interaction between those involved in service delivery and users in primary and secondary care. With small to medium size laboratories facing potential contracting out or rationalisation within larger purchasing authorities, there is an increasing need to establish criteria to ensure continued delivery of a high standard diagnostic service. Rationalisation needs to ensure that proposed alternative ways of service delivery need to achieve at least the same local standards of quality, if not better, at the appropriate level of use of resources. Such standards of service delivery could include assessment of: performance in internal as well as external quality assessment schemes; the need for locally based consultative input and advice; turnround time; staff skills and training; technology used; and the level of interaction required by users.

There is a lack of independent professional advice for input at local unit level into these exercises. Such input needs to have the respect and confidence of users, purchasers, general managers and service providers. In the absence of such advisory machinery, management consultants and health economists are increasingly involved in such assessment of option appraisals, as Trusts are caught in the fervour of market testing. This trend of market testing may become widespread, particularly with the lack of financial dividends from the resource management initiative and the uncertain future of internal trading, both of which it was hoped would check the level of use of diagnostic and other services.

There is nothing wrong with trying to assess whether a service is providing value for money. (Many would probably suggest that administration, information and finance departments are ideally suited for market testing.) However, sound advice needs to be made available, to ensure that standards of quality are upheld and input is maintained from users of the service as wel as provider managers and purchasers. Otherwise, the loss of one clinical service may signal danger for other clinical services, whenever offsite provision is seen as a plausible and an attractive option.

$$
\begin{array}{r}
\text { A WAISE } \\
\text { Department of Chemical Pathology, } \\
\text { Friarage Hospital, } \\
\text { North Yorkshire DL6 } 19 G
\end{array}
$$

1 NHS Management Executive. EL(93)37 White Paper-Competing for Quality market testing and reporting. London: Department of testing and reponith, 1993 .

2 Tew CJ. Selling of the pathology service. Bull $R$ Coll Pathol 1993;81:23.

3 Scottish Health Service Advisory Council Specifications of laboratory services: Guideline for general managers. Edinburgh: Scottish Office, 1992

\section{Selective medium for MRSA}

We read with great interest the article by Wood and colleagues in which they evaluated aztreonam blood agar as a selective medium for Gram positive aerobic bacteria. ${ }^{1}$ MRSA Selectatabs are commercial (MAST) media supplements containing oxacillin (final concentration $4 \mathrm{mg} / \mathrm{l}$ ) used in the detection of methicillin resistant Staphylococcus aureus (MRSA). We recently evaluated this product and concluded, along with Lally and colleagues, ${ }^{2}$ that oxacillin could be used optimally when incorporated into Mannitol Salt Agar (MSA). This permits simultaneous detection and presumptive identification of MRSA on media incubated at $37^{\circ} \mathrm{C}$. The recent isolation of a strain of MRSA prompted a large surveillance operation. We used MSA (Oxoid) containing methicillin $(5 \mathrm{mg} / \mathrm{l})$ and aztreonam $(10 \mathrm{mg} / \mathrm{l})$ to $\operatorname{good}$ effect and commend its use to our colleagues.

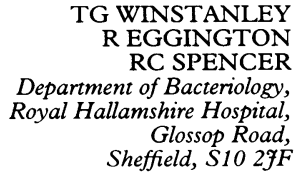

Wood W, Harvey G, Olson ES, Reid TMS Aztreonam selective agar for Gram positive bacteria. F Clin Pathol 1993;46:769-71.

2 Lally RT, Ederer MN, Woolfrey BF. Evaluation of mannitol salt agar with oxacillin as a screening medium for methicillin-resistant Staphylococcus aureus. $\mathcal{f}$ Clin Microbiol 1985;22:501-4. 